\title{
Nonlinear Model Predictive Control Considering Stochastic and Systematic Uncertainties with Sets of Densities
}

\author{
Achim Hekler, Daniel Lyons, Benjamin Noack, and Uwe D. Hanebeck
}

\begin{abstract}
In Model Predictive Control, the quality of control is highly dependent upon the model of the system under control. Therefore, a precise deterministic model is desirable. However, in real-world applications, modeling accuracy is typically limited and systems are generally affected by disturbances. Hence, it is important to systematically consider these uncertainties and to model them correctly. In this paper, we present a novel Nonlinear Model Predictive Control method for systems affected by two different types of perturbations that are modeled as being either stochastic or unknown but bounded quantities. We derive a formal generalization of the Nonlinear Model Predictive Control principle for considering both types of uncertainties simultaneously, which is achieved by using sets of probability densities. In doing so, a more robust and reliable control is obtained. The capabilities and benefits of our approach are demonstrated in real-world experiments with miniature walking robots.
\end{abstract}

\section{INTRODUCTION}

In recent years, Model Predictive Control (MPC), which is also called Receding or Rolling Horizon Control, gained increasing interest for control applications from various fields. In contrast to regular control, MPC does not only consider the current state of the system under control, but also its future dynamic behavior. By model-based prediction of future system states, it is possible to react earlier to the anticipated development of the system and thus, a higher quality of control can be achieved.

Even though the incorporation of uncertainties leads to a significant increase in quality of control, most MPC approaches do not explicitly consider the influence of noise on the system [1]. In real-world applications, the controlled systems are generally affected by disturbances, caused by an uncertain state estimation, exogenous influences, or modeling errors. In order to achieve a robust Model Predictive Control in the presence of these unknown quantities, it is important to systematically consider these uncertainties and to model them correctly.

Consideration of uncertainties in the optimal control problem has traditionally been treated in a stochastic way, cf. [2], [3], and [4]. Therefore, all disturbances affecting the system are described by random variables characterized by the underlying probability density functions. The objective of control is to maximize the expected rewards of the predicted states, obtained by applying the control sequence.

Achim Hekler, Daniel Lyons, Benjamin Noack, and Uwe D. Hanebeck are with the Intelligent Sensor-Actuator-Systems Laboratory (ISAS), Karlsruhe Institute of Technology (KIT), Germany. Email: \{achim.hekler|lyons|noack\}@kit.edu, uwe.hanebeck@ieee.org.

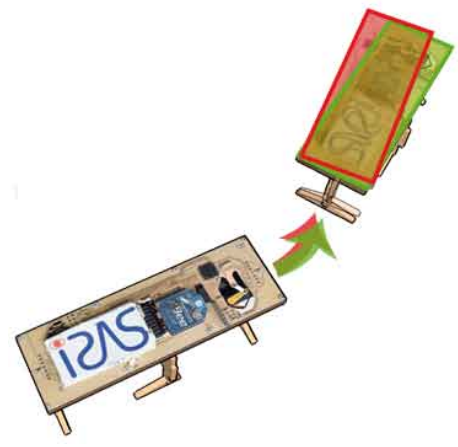

Fig. 1. Schematic left turn of a miniature walking robot. The light (green) labeled robot represents the theoretically intended movement, the dark (red) one is the result subject to real-world conditions with errors.

In many practical applications, the characteristics of the perturbations may not be known in detail, but can be assumed to belong to a bounded set. In addition, the system can also be affected by errors, which do not have a probabilistic nature or which we cannot model sufficiently precise in a stochastic manner. Thus, these uncertain quantities can be modeled far better by their membership to a given set rather than by a probabilistic description [5], [6], [7], [8], and [9]. The most popular set-membership approaches, where the sets admit a simple parametrical characterization, are polyhedral and ellipsoidal descriptions of the error [2]. In both cases, the predicted states of a linearized system can also be modeled by a polyhedron or an ellipsoid, respectively.

These existing methods always model disturbances either in a purely probabilistic or in a purely set-valued manner. However, in real-world applications, both kinds of errors affect the system at the same time. Thus, these purely probabilistic or purely set-valued models impose a specific uncertainty representation on each disturbance, even if its natural characteristics deviate severely from this representation. In this paper, we introduce a novel approach combining both probabilistic and systematic uncertainties by using sets of densities. In doing so, a more sophisticated description of uncertain quantities and accordingly of the whole system under control is possible. This results in a more realistic prediction of the future development of the system and, hence, a more robust and reliable control is achievable.

This procedure is different from methods such as [10] or [11], which model the uncertainties exclusively in a stochastic, but not in a set-valued way. These approaches 
use set-membership to ensure soft and hard constraints on stochastic states for a robust control.

The paper is structured as follows: In the next section, the considered MPC problem is described. In Section III, the prediction step of a generalized Extended Kalman Filter (EKF) incorporating both probabilistic and systematic noise is presented. By using this prediction method in a Model Predictive Controller, conventional stochastic or set-valued reward functions are no longer directly applicable. Therefore, a generalization of existing approaches will be introduced in Section IV. In Section V, we present the results of a realworld experiment, in which the methods of our proposed approach are compared to an EKF-based MPC. An outlook to future work will conclude this paper.

\section{PRoblem Formulation}

We present a novel approach for Nonlinear Model Predictive Control (NMPC) of systems that are affected by different disturbances. These disturbances will be modeled not only as stochastic, but also as set-valued quantities.

The dynamic behavior of the system is described by a nonlinear discrete-time system of the form

$$
\underline{\boldsymbol{x}}_{k+1}=\underline{a}_{k}\left(\underline{\boldsymbol{x}}_{k}, \underline{u}_{k}, \underline{\boldsymbol{w}}_{k}, \underline{d}_{k}\right),
$$

where underlined letters denote vectors and random vectors are written in boldface letters. The possibly time-variant system function $\underline{a}_{k}(\cdot)$ maps the inaccessible vector-valued system state $\underline{\boldsymbol{x}}_{k} \in \mathbb{R}^{n}$ to the state $\underline{\boldsymbol{x}}_{k+1}$ at time step $k+1$. Furthermore, $\underline{u}_{k}$ denotes the applied control input taken from a discrete set $\mathcal{U}_{k}$.

The random variable $\underline{\boldsymbol{w}}_{k}$ represents those errors that we model stochastically, characterized by a probability density function $f_{k}^{w}$, and $\underline{d}_{k} \in \mathcal{D}_{k} \subseteq \mathbb{R}^{n}$ denotes the errors that will be modeled as systematic disturbances. There is no more knowledge about $\underline{d}_{k}$ than its membership to a known bounded set. In particular, $\underline{d}_{k}$ does not have to be constant over time. Hence, deriving an appropriate model for the evolution of $\underline{d}_{k}$ can turn out to be an elaborate task. Additionally, an explicit estimation of $\underline{d}_{k}$ by means of a disturbance observer generally suffers from slow convergence rates especially when $\underline{d}_{k}$ is subject to abrupt changes over time. Thus, especially when reliable results are instantly required, it is not suitable to estimate the unknown bias by means of state augmentation.

Based on the system equation (1), the Model Predictive Control problem is to find an optimal sequence $\underline{u}_{k: k+(N-1)}^{*}=\left[\underline{u}_{k}^{*}, \ldots, \underline{u}_{k+(N-1)}^{*}\right]$ of control inputs. Optimality is defined in terms of maximizing a cumulative reward function $V_{k}\left(\underline{\boldsymbol{x}}_{k: k+N}, \underline{u}_{k: k+(N-1)}\right)$, giving rewards over a time horizon of length $N$ [2]. The first control input $\underline{u}_{k}^{*}$ from the sequence $\underline{u}_{k: k+(N-1)}^{*}$ is then applied to the system. For details on the cumulative reward function, see Section IV.

\section{Example System: System Equation}

For the purpose of illustration, we consider throughout the whole paper the specific example of a mobile miniature walking robot (Fig. 1) moving on a 2D-plane with constant velocity. This omnidirectional robot is used in a specific mode of operation, where left and right turns are superimposed onto the forward motion [12]. Similar to the motion of twowheeled differential-drive vehicles, the motion can be modeled by means of a nonlinear discrete-time system equation

$$
\underline{\boldsymbol{x}}_{k+1}=\left[\begin{array}{c}
\boldsymbol{x}_{k+1} \\
\boldsymbol{y}_{k+1} \\
\boldsymbol{\phi}_{k+1}
\end{array}\right]=\left[\begin{array}{c}
\boldsymbol{x}_{k} \\
\boldsymbol{y}_{k} \\
\boldsymbol{\phi}_{k}
\end{array}\right]+\left[\begin{array}{c}
T \cdot \cos \left(\boldsymbol{\phi}_{k}\right) \\
T \cdot \sin \left(\phi_{k}\right) \\
u_{k}
\end{array}\right]+\left[\begin{array}{c}
\boldsymbol{w}_{k}^{x} \\
\boldsymbol{w}_{k}^{y} \\
\boldsymbol{w}_{k}^{\phi}
\end{array}\right],
$$

where $\boldsymbol{x}_{k}$ and $\boldsymbol{y}_{k}$ are the coordinates of the position and $\phi_{k}$ is the orientation of the robot. Furthermore, $T$ denotes the constant step size and $\boldsymbol{w}_{k}$ the noise influence on the system. The input $u_{k}$ is a steering action, i.e., a change of direction of the robot, chosen from the discrete set

$$
\mathcal{U}_{k}=\left\{0^{\circ}, \pm 2^{\circ}, \pm 4^{\circ}\right\} \text {. }
$$

In real applications, movement of the robots is imprecise and despite individual calibration it cannot be ensured that the chosen angle is applied correctly. Because this error does not have a probabilistic manner, it is more suitable to model it as a systematic error instead of modeling it stochastically. By assuming that the systematic error $d_{k}$ only affects the steering angle directly, the system equation is given by

$$
\left[\begin{array}{l}
\boldsymbol{x}_{k+1} \\
\boldsymbol{y}_{k+1} \\
\boldsymbol{\phi}_{k+1}
\end{array}\right]=\left[\begin{array}{l}
\boldsymbol{x}_{k} \\
\boldsymbol{y}_{k} \\
\boldsymbol{\phi}_{k}
\end{array}\right]+\left[\begin{array}{c}
T \cdot \cos \left(\boldsymbol{\phi}_{k}\right) \\
T \cdot \sin \left(\boldsymbol{\phi}_{k}\right) \\
u_{k}
\end{array}\right]+\left[\begin{array}{c}
\boldsymbol{w}_{k}^{x} \\
\boldsymbol{w}_{k}^{y} \\
\boldsymbol{w}_{k}^{\phi}
\end{array}\right]+\left[\begin{array}{c}
0 \\
0 \\
d_{k}
\end{array}\right] .
$$

III. Stochastic and Set-Valued State Prediction WITH SETS OF DENSITIES

In order to allow for the simultaneous treatment of stochastic and systematic uncertainties, we propose a generalization of classical probability theory. In the following, the combination of a stochastic and a set-valued quantity will turn out to be characterized by a set of probability densities.

\section{A. Generic State Prediction with Sets of Densities}

In terms of the underlying probability density functions, the system dynamics (1) can also be expressed by the transition density

$$
\begin{aligned}
& f_{k}^{\mathrm{T}}\left(\underline{x}_{k+1} \mid \underline{x}_{k}, \underline{\hat{u}}_{k}, \underline{d}_{k}\right) \\
& \quad=\int_{\mathbb{R}^{n}} \delta\left(\underline{x}_{k+1}-\underline{a}_{k}\left(\underline{x}_{k}, \underline{\hat{u}}_{k}, \underline{w}_{k}, \underline{d}_{k}\right)\right) f_{k}^{w}\left(\underline{w}_{k}\right) d \underline{w}_{k},
\end{aligned}
$$

where $\delta$ denotes the $n$-dimensional Dirac delta function. For concrete $\underline{\hat{u}}_{k}$ and $\underline{d}_{k}$, the probability density function $f_{k+1}^{\mathrm{p}}$ of $\underline{\boldsymbol{x}}_{k+1}$ can then be computed from the Chapman-Kolmogorov equation

$$
f_{k+1}^{\mathrm{p}}\left(\underline{x}_{k+1}\right)=\int_{\mathbb{R}^{n}} f_{k}^{\mathrm{T}}\left(\underline{x}_{k+1} \mid \underline{x}_{k}, \underline{\hat{u}}_{k}, \underline{d}_{k}\right) f_{k}^{\mathrm{p}}\left(\underline{x}_{k}\right) d \underline{x}_{k} .
$$

However, due to the unknown but bounded quantity $\underline{d}_{k} \in \mathcal{D}_{k}$, the state transition is not unique. Thus, in contrast to a purely stochastic modeling, one has to consider the whole set of possible transition densities

$$
\mathcal{F}_{k}^{\mathrm{T}}=\left\{f_{k}^{\mathrm{T}}\left(\underline{x}_{k+1} \mid \underline{x}_{k}, \underline{\hat{u}}_{k}, \underline{d}_{k}\right) \mid \underline{d}_{k} \in \mathcal{D}_{k}\right\} .
$$

Consequently, equation (4) has to be calculated elementwise for every $f_{k}^{\mathrm{T}} \in \mathcal{F}_{k}^{\mathrm{T}}$ and a set $\mathcal{F}_{k+1}^{\mathrm{p}}$ of predicted densities is obtained. For the subsequent time update, this set will then be processed elementwise with $\mathcal{F}_{k+1}^{\mathrm{T}}$. 
Obviously, the uncertain state $\underline{\boldsymbol{x}}_{k}$ is not characterized by a single probability density anymore, but rather by a set of densities. While focussing on convex sets of functions, this way of generalizing classical probability theory has been studied in [13]. Also, approaches to Bayesian state estimation such as [14] and [15] are based on sets of densities. All of these concepts are aimed at providing wider possibilities of modeling uncertainty in order to overcome the limitations of a purely stochastic point of view.

Without utilizing appropriate approximation techniques, the prediction step (4) is in general infeasible to compute, even when dealing with unique densities, i.e., in the purely stochastic case. So using sets of arbitrary densities aggravates this issue. For the important special case of linear system dynamics perturbed by Gaussian noise, the prediction step with sets of densities can be expressed in closed form, as it will be shown in the following section.

\section{B. Linear State Prediction with Ellipsoidal Sets of Means}

Let the initial state $\underline{x}_{0}$ be characterized by a Gaussian density $\mathcal{N}\left(\underline{\hat{x}}_{0}^{\mathrm{p}}, \mathbf{C}_{0}^{\mathrm{p}}\right)$ with mean $\underline{\hat{x}}_{0}^{\mathrm{p}}$ and covariance matrix $\mathbf{C}_{0}^{\mathrm{p}}$. By assuming that the system function (1) can be rewritten as a linear mapping

$$
\underline{\boldsymbol{x}}_{k+1}=\mathbf{A}_{k} \underline{\boldsymbol{x}}_{k}+\mathbf{B}_{k}\left(\underline{\hat{u}}_{k}+\underline{\boldsymbol{w}}_{k}+\underline{d}_{k}\right)
$$

and that $\underline{\boldsymbol{w}}_{k}$ is a zero-mean Gaussian white noise $\mathcal{N}\left(\underline{0}, \mathbf{C}_{k}^{w}\right)$, the predicted density $f_{k+1}^{\mathrm{p}}$ for a certain $\underline{d}_{k}$ will become also a Gaussian density

$$
f_{k+1}^{\mathrm{p}}=\mathcal{N}\left(\underline{\hat{x}}_{k+1}^{\mathrm{p}}, \mathbf{C}_{k+1}^{\mathrm{p}}\right)
$$

with mean

$$
\underline{\hat{x}}_{k+1}^{\mathrm{p}}=\mathbf{A}_{k} \underline{\hat{x}}_{k}^{\mathrm{p}}+\mathbf{B}_{k}\left(\underline{\hat{u}}_{k}+\underline{d}_{k}\right)
$$

and covariance matrix

$$
\mathbf{C}_{k+1}^{\mathrm{p}}=\mathbf{A}_{k} \mathbf{C}_{k}^{\mathrm{p}} \mathbf{A}_{k}^{\mathrm{T}}+\mathbf{B}_{k} \mathbf{C}_{k}^{w} \mathbf{B}_{k}^{\mathrm{T}}
$$

Apparently, the unknown but bounded vector $\underline{d}_{k} \in \mathcal{D}_{k}$ only affects the computation of the expected value $\underline{\hat{x}}_{k+1}^{\mathrm{p}}$. Therefore, equation (5) turns into a Minkowski sum

$$
\mathcal{X}_{k+1}^{\mathrm{p}}=\mathbf{A}_{k} \mathcal{X}_{k}^{\mathrm{p}} \oplus \mathbf{B}_{k} \mathcal{U}_{k}
$$

which is the elementwise summation of $\mathbf{A}_{k} \mathcal{X}_{k}^{\mathrm{p}}$ and

$$
\mathbf{B}_{k} \mathcal{U}_{k}=\left\{\mathbf{B}_{k}\left(\underline{\hat{u}}_{k}+\underline{d}_{k}\right) \mid \underline{d}_{k} \in \mathcal{D}_{k}\right\} .
$$

Finally, the predicted state $\underline{\boldsymbol{x}}_{k+1}$ is characterized by a set

$$
\mathcal{F}_{k+1}^{\mathrm{p}}=\left\{\mathcal{N}\left(\underline{\hat{x}}_{k+1}^{\mathrm{p}}, \mathbf{C}_{k+1}^{\mathrm{p}}\right) \mid \underline{\hat{x}}_{k+1}^{\mathrm{p}} \in \mathcal{X}_{k+1}^{\mathrm{p}}\right\}
$$

of translated Gaussian densities with identical covariance matrices.

This concept of incorporating systematic bounded uncertainties rests upon the derivations in [16]. Therein, ellipsoidal sets are utilized to represent $\mathcal{X}_{k}^{\mathrm{p}}$ and $\mathcal{U}_{k}$. An ellipsoid

$$
\mathcal{E}(\underline{\hat{c}}, \mathbf{X}):=\left\{\underline{x} \in \mathbb{R}^{n} \mid(\underline{x}-\underline{\hat{c}})^{\mathrm{T}} \mathbf{X}^{-1}(\underline{x}-\underline{\hat{c}}) \leq 1\right\}
$$

is parameterized by a midpoint $\underline{\hat{c}} \in \mathbb{R}^{n}$ and a nonnegative definite shape matrix $\mathbf{X} \in \mathbb{R}^{n \times n}$. In particular, this representation facilitates the calculation of an affine transformation

$$
\mathbf{A} \mathcal{E}(\underline{\hat{c}}, \mathbf{X})+\underline{b}=\mathcal{E}\left(\mathbf{A} \underline{\hat{c}}+\underline{b}, \mathbf{A X} \mathbf{A}^{\mathrm{T}}\right) .
$$

The Minkowski sum of two ellipsoid does not in general result in an ellipsoid anymore. An easy way to approximate a Minkowski sum by an enclosing ellipsoid is given by the inclusion

$$
\mathcal{E}\left(\underline{\hat{c}}_{1}, \mathbf{X}_{1}\right) \oplus \mathcal{E}\left(\underline{\hat{c}}_{2}, \mathbf{X}_{2}\right) \subseteq \mathcal{E}\left(\underline{\hat{c}}_{1}+\underline{\hat{c}}_{2}, \mathbf{X}(p)\right)
$$

with

$$
\mathbf{X}(p)=\left(1+p^{-1}\right) \mathbf{X}_{1}+(1+p) \mathbf{X}_{2}
$$

for any $p>0$ [9]. An outer approximation with minimal sum of squares of semiaxes, i.e., minimal trace of $\mathbf{X}(p)$, is obtained for

$$
p=\frac{\operatorname{trace}\left(\mathbf{X}_{1}\right)^{\frac{1}{2}}}{\operatorname{trace}\left(\mathbf{X}_{2}\right)^{\frac{1}{2}}} .
$$

This short excursion to ellipsoidal calculus provides the groundwork for efficiently evaluating equation (7). With $\mathcal{X}_{k}^{\mathrm{p}}=\mathcal{E}\left(\underline{\hat{c}}_{k}^{\mathrm{p}}, \mathbf{X}_{k}^{\mathrm{p}}\right)$ and $\mathcal{U}_{k}=\mathcal{E}\left(\underline{\hat{u}}_{k}, \mathbf{U}_{k}\right)$, equation (7) then becomes

$$
\begin{aligned}
\mathcal{X}_{k+1}^{\mathrm{p}} & \stackrel{\text { eq. (7) }}{=} \mathbf{A}_{k} \mathcal{E}\left(\underline{\hat{c}}_{k}^{\mathrm{p}}, \mathbf{X}_{k}^{\mathrm{p}}\right) \oplus \mathbf{B}_{k} \mathcal{E}\left(\underline{\hat{u}}_{k}, \mathbf{U}_{k}\right) \\
& \stackrel{\text { eq. (8) }}{=} \mathcal{E}\left(\mathbf{A}_{k} \underline{\hat{c}}_{k}^{\mathrm{p}}, \mathbf{A}_{k} \mathbf{X}_{k}^{\mathrm{p}} \mathbf{A}_{k}^{\mathrm{T}}\right) \oplus \mathcal{E}\left(\mathbf{B}_{k} \underline{\hat{u}}_{k}, \mathbf{B}_{k} \mathbf{U}_{k} \mathbf{B}_{k}^{\mathrm{T}}\right) \\
& \stackrel{\text { eq. (9) }}{\subseteq} \mathcal{E}\left(\underline{\hat{c}}_{k+1}^{\mathrm{p}}, \mathbf{X}_{k+1}^{\mathrm{p}}\right)
\end{aligned}
$$

with

$$
\underline{\hat{c}}_{k+1}^{\mathrm{p}}=\mathbf{A}_{k} \underline{\hat{c}}_{k}^{\mathrm{e}}+\mathbf{B}_{k} \underline{\hat{u}}_{k}
$$

and

$$
\mathbf{X}_{k+1}^{\mathrm{p}}=\left(1+p^{-1}\right) \mathbf{A}_{k} \mathbf{X}_{k}^{\mathrm{e}} \mathbf{A}_{k}^{\mathrm{T}}+(1+p) \mathbf{B}_{k} \mathbf{U}_{k} \mathbf{B}_{k}^{\mathrm{T}},
$$

where the optimal $p$ is calculated from equation (10). Thus, $\underline{\boldsymbol{x}}_{k+1}$ is characterized by the set

$$
\mathcal{F}_{k+1}^{\mathrm{p}}=\left\{\mathcal{N}\left(\underline{\hat{x}}_{k+1}^{\mathrm{p}}, \mathbf{C}_{k+1}^{\mathrm{p}}\right) \mid \underline{\hat{x}}_{k+1}^{\mathrm{p}} \in \mathcal{E}\left(\underline{\hat{c}}_{k+1}^{\mathrm{p}}, \mathbf{X}_{k+1}^{\mathrm{p}}\right)\right\}
$$

of Gaussian densities. Obviously, this predicted set of Gaussian densities is simply parameterized by the corresponding ellipsoid $\mathcal{E}\left(\hat{\hat{c}}_{k+1}^{\mathrm{p}}, \mathbf{X}_{k+1}^{\mathrm{p}}\right)$ of means and the covariance matrix $\mathbf{C}_{k+1}^{\mathrm{p}}$, which are calculated from (6) and (11), respectively. Compared to the purely stochastic linear state prediction, the presented concept only differs in one additional matrix calculation, namely equation (13).

\section{Nonlinear State Prediction with Ellipsoidal Sets of Means}

In order to adapt this concept to nonlinear systems, a linearization of the system function (1) can be performed. The typical first-order Taylor series expansion around the predicted mean - as it is done within the EKF framework - is inappropriate, since there is no unique mean anymore. Therefore, a linearization over the entire set of means has to be determined. A way to find such a linearization has been discussed in [16] and [17]. Here, the ellipsoid of means is 
approximated by a fixed number of equidistant points on the principal axes. A linearization for the entire set of means is then determined by employing a least-squares fit.

\section{NMPC WITH SETS OF DENSITIES}

The Nonlinear Model Predictive Controller aims at maximizing an application-specific cumulative reward over a fixed time horizon. The cumulative reward function is calculated by predicting the impact of control inputs on the future development of the system and rating this development at every time instant by successively evaluating one-step reward functions. At every time step $k$, an open-loop optimal control problem is solved for a time horizon of length $N$.

In the deterministic case, the system state and a control input $\underline{u}_{k}$ are rated by the scalar output of a one-step reward function

$$
g_{k}^{\operatorname{det}}\left(\underline{x}_{k}, \underline{u}_{k}\right)
$$

If the system state is described by a random variable $\underline{x}_{k}$, characterized by a probability density $f_{k}$, the one-step reward function

$$
g_{k}^{\text {stoc }}\left(\underline{\boldsymbol{x}}_{k}, \underline{u}_{k}\right)
$$

is a scalar function of the random variable $\underline{x}_{k}$. It can be defined, for example, as the expected value

$$
\begin{aligned}
g_{k}^{\text {stoc }}\left(\underline{\boldsymbol{x}}_{k}, \underline{u}_{k}\right) & =\mathrm{E}_{\underline{\boldsymbol{x}}_{k}}\left(g_{k}^{\operatorname{det}}\left(\underline{x}_{k}, \underline{u}_{k}\right)\right) \\
& =\int g_{k}^{\operatorname{det}}\left(\underline{x}_{k}, \underline{u}_{k}\right) f_{k}\left(\underline{x}_{k}\right) d \underline{x}_{k}
\end{aligned}
$$

of a deterministic one-step reward function as in (14) over the random variable $\underline{x}_{k}$.

From such a one-step reward function, the cumulative reward over a certain time horizon $N$ is given by

$$
\begin{aligned}
V & \left(\underline{\boldsymbol{x}}_{k: k+N}, \underline{u}_{k: k+N-1}\right) \\
\quad & =\max _{\underline{u}_{k: k+N-1}}\left\{g_{k+N}^{\text {stoc }}\left(\underline{\boldsymbol{x}}_{k+N}\right)+\sum_{i=k}^{k+N-1} g_{i}^{\text {stoc }}\left(\underline{\boldsymbol{x}}_{i}, \underline{u}_{i}\right)\right\},
\end{aligned}
$$

which maximizes rewards over $N$ time steps with terminal reward $g_{k+N}^{\text {stoc }}\left(\underline{\boldsymbol{x}}_{k+N}\right)$.

We now generalize this concept to random variables $\underline{\boldsymbol{x}}_{k}$ characterized by sets $\boldsymbol{\mathcal { F }}_{k}$ of probability densities. The extension of the stochastic reward function in (15) is defined as

$$
\mathcal{G}_{k}\left(\underline{\boldsymbol{x}}_{k}, \underline{u}_{k}\right):=\left\{g_{k}^{\text {stoc }}\left(\underline{\boldsymbol{x}}_{k}, \underline{u}_{k}\right) \mid \underline{\boldsymbol{x}}_{k} \sim f_{k} \text { with } f_{k} \in \mathcal{F}_{k}\right\} .
$$

in a straightforward manner. This means that the stochastic one-step reward function $g_{k}^{\text {stoc }}$ is evaluated for every probabilistic characterization of $\underline{\boldsymbol{x}}_{k}$, i.e., for all possible densities in $\mathcal{F}_{k}$. In doing so, we obtain a set $\mathcal{G}_{k}$ of one-step rewards. For the one-step reward function in (16), this results in the set

$$
\mathcal{G}_{k}\left(\underline{\boldsymbol{x}}_{k}, \underline{u}_{k}\right):=\left\{\int g_{k}^{\mathrm{det}}\left(\underline{x}_{k}, \underline{u}_{k}\right) f_{k}\left(\underline{x}_{k}\right) d \underline{x}_{k} \mid f_{k} \in \mathcal{F}_{k}\right\}
$$

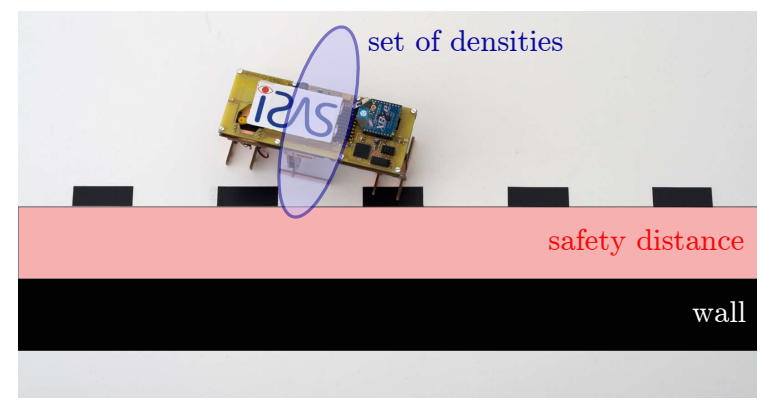

Fig. 2. Setup of the real-world experiment. The walking robot moves along a wall in a safety distance. Future system states are judged by the distance of the ellipsoid of means to the wall.

of expected rewards. By applying the max-min principle known from purely set-valued approaches [2], we take the minimal reward

$$
g_{k}\left(\underline{\boldsymbol{x}}_{k}, \underline{u}_{k}\right):=\inf \left\{\mathcal{G}_{k}\left(\underline{\boldsymbol{x}}_{k}, \underline{u}_{k}\right)\right\}
$$

received from all elements in $\mathcal{F}_{k}$. This combined one-step reward function $g_{k}$ maps a set of densities to a well-defined scalar value and can be used for the calculation of the cumulative reward function (17) over a fixed time horizon. We have thus achieved a combination of stochastic and setvalued control.

\section{Example System: Reward Function}

The considered control objective for the miniature walking robot is to move along a wall while keeping a safety distance without crashing into the wall [18]. For a formulation of a combined reward function for this specific scenario, we will closely follow the layout presented above.

In a deterministic setup, one possible reward function would be the distance of the robot to the wall. The stochastic reward constructed from this deterministic one could be the distance of the wall to the mean of the current state estimate of the position. If we now incorporate unknown but bounded uncertainties into the state estimation and, hence, into control, the stochastic reward function would be calculated for every possible density in the set $\mathcal{F}_{k}$. The resulting combined one-step reward function would be the minimum of these distances. For more details, we refer the reader to the next section.

\section{EXPERIMENTS}

In order to illustrate the capabilities of the novel NMPC approach as well as the benefits resulting from considering stochastic and systematic errors, several test runs under real-world conditions with the walking robots introduced in Section II were conducted. For benchmarking the results of these experiments, we compare our approach to one based on the widely used EKF, where the bias is estimated by means of a disturbance observer and is supposed to be piecewise constant.

\section{A. Description of the Test Environment}

The walking robots are part of a test environment built to evaluate control and localization methods [12]. For control purposes, an overhead camera overlooks a confined area, in which the robots move, and determines the true postions of 
the robots. Both the experiments with real robots as well as simulations can be controlled and monitored by a graphical user interface (GUI). The GUI shows the true positions of the robots as well as their estimated positions.

\section{B. Scenario}

In the selected scenario, the task of the walking robot was to move as accurately as possible along a wall in a straight line with a safety distance of $5 \mathrm{~cm}$ as depicted in Fig. 2. Since the system state (2) of the robot is not directly accessible, its current state has to be estimated by means of dead reckoning and noisy distance measurements to three landmarks. In order to incorporate the systematic errors acting on the robots, we employed the approach described in [16] for state estimation. In contrast to [16], we assume only stochastic noise for the emulated distance measurements modeled by the nonlinear equation

$$
\boldsymbol{z}_{k}=\sqrt{\left(\boldsymbol{x}_{k}-\check{x}_{k}^{i}\right)^{2}+\left(\boldsymbol{y}_{k}-\check{y}_{k}^{i}\right)^{2}}+\boldsymbol{v}_{k},
$$

where $\left[\check{x}_{k}^{i}, \check{y}_{k}^{i}\right]^{\mathrm{T}}$ is the two-dimensional position of landmark $i=1,2,3$ and $\boldsymbol{v}_{k}$ white Gaussian measurement noise.

At each time instant, a myopic open-loop control problem is solved and the optimal control input is applied to the system. Due to the linearized system and measurement equation, the considered sets of densities consist of Gaussians with identical covariance matrices. Since the systematic disturbances affecting the system are modeled as an ellipsoid, each set of Gaussians can be parameterized by the corresponding ellipsoid $\mathcal{E}\left(\underline{\hat{c}}_{k}, \mathbf{X}_{k}\right)$ of means (see Section III-C). The chosen combined reward function is

$$
g_{k}\left(\underline{\boldsymbol{x}}_{k}, u_{k}\right)=-\left(d\left(\mathcal{E}\left(\underline{\hat{c}}_{k}, \mathbf{X}_{k}\right), \mathcal{W}\right)^{2}+\alpha \cdot\left|\hat{\phi}_{k}\right|\right)
$$

where $\mathcal{W}$ is the set of all points of the wall and $d(\cdot, \cdot)$ is defined as

$$
\begin{aligned}
& d\left(\mathcal{E}\left(\underline{\hat{c}}_{k}, \mathbf{X}_{k}\right), \mathcal{W}\right) \\
& =\left\{\begin{array}{cl}
\min _{\substack{\underline{x}_{k} \in \mathcal{E}\left(\hat{c}_{k}, \mathbf{x}_{k}\right) \\
\underline{y} \in \mathcal{W}}}\left\|\underline{x}_{k}-\underline{y}\right\| & \text { if }\left\|\underline{x}_{k}-\underline{y}\right\| \geq 5 \mathrm{~cm} \\
C & \text { otherwise }
\end{array}\right.
\end{aligned}
$$

with $C$ an arbitrarily large number and $\|\cdot\|$ the Euclidean norm. So $d\left(\mathcal{E}\left(\underline{\hat{c}}_{k}, \mathbf{X}_{k}\right), \mathcal{W}\right)$ is the minimal distance between the ellipsoid $\mathcal{E}\left(\underline{\hat{c}}_{k}, \mathbf{X}_{k}\right)$ and the wall, if this distance is larger than the safety distance as depicted in Fig. 2. If the distance of at least one point of the ellipsoid to the wall is less than the safety distance, we penalize this by specifying a high negative reward $C$. The parameter $\hat{\phi}_{k}$ in the above equation is the expectation of the orientation of the robot over the Gaussian with mean $\underline{\hat{c}}_{k}$. This Gaussian corresponds to the center of the ellipsoid $\mathcal{E}\left(\underline{\hat{c}}_{k}, \mathbf{X}_{k}\right)$. To adjust the smoothness of the trajectory, the factor $\alpha$ allows a weighting of the orientation of the robot.

\section{Experimental Setup}

For all test runs, we selected the following parameters. The step size of the robot is constant $T=10 \mathrm{~mm}$. The probabilistic noise influences on the system $w_{k}^{x}, w_{k}^{y}$, and $w_{k}^{\phi}$ are assumed to be zero-mean white Gaussian noises with standard deviations $\sigma_{w}^{x}=0.2 \mathrm{~mm}, \sigma_{w}^{y}=0.2 \mathrm{~mm}$, and $\sigma_{w}^{\phi}=0.2 \mathrm{rad}$. The initial probabilistic uncertainties in both dimensions of the position are $\sigma_{w}^{x, 0}=\sigma_{w}^{y, 0}=20 \mathrm{~mm}$ and the uncertainty in its orientation $\sigma_{w}^{\phi, 0}=0.02 \mathrm{rad}$. The initial setvalued bounds are $10 \mathrm{~mm}$ in both dimensions of the position. Furthermore, measurements are taken to the three landmarks after each seventh step. In order to guarantee comparability, we position the robot at the same coordinate of the arena for every test run. Also the positions of the landmarks remain the same.

\section{Results}

The results of the experiments applying the proposed approach are compared with an EKF-based NMPC method, where the state is augmented by the unknown bias. The dynamic behavior of this bias is modeled by a piecewise constant function. The EKF-based controller employs the same reward function as in (18) but instead of using $d(\mathcal{E}(\underline{\hat{c}}, \mathbf{X}), \mathcal{W})$, we take the distance of the expected value to the wall. Two exemplary trajectories are depicted in Fig. 3.

The experiments clearly show that the proposed controller is more robust. The robot always stays in a safety distance to the wall while the EKF-based controller leads to very close distances to the wall or even crashes. This poor behavior results from the slow convergence rate of the estimate of the bias, especially when it changes abruptly. Furthermore, the path of the combined controller is smoother than the path of the EKF-based controller. Although being a little bit more conservative, the combined controller leads to a safer and smoother trajectory. The same results cannot be achieved with the EKF-based controller by simply selecting a larger safety distance, because the influence of drift on state estimation cannot be evaluated in advance and hence one does not know how large the safety distance has to be chosen. The results also show that the used localization method is adequate for systems affected by systematic and stochastic noise. Fig. 3 (a) demonstrates that the true position of the robot always lies in the estimated set of densities. The set does not diverge over time, as it gets smaller with every measurement step.

\section{CONClusions And Future Work}

We introduced a new combined stochastic and set-valued approach for Model Predictive Control by defining a reward function that does not only regard stochastic disturbances but also systematic errors. This novel reward function is obtained by the formal generalization of stochastic reward functions to sets of densities. By employing this technique, a more robust and reliable control is achievable as we demonstrated in real-world experiments.

Future work will be concerned with the extension of this method by additionally incorporating properties of the measurement process in the planning procedure as in [19]. This allows to consider systematic errors not only in the system model but also systematic measurement errors as they often occur in real-world applications. It is also of interest 

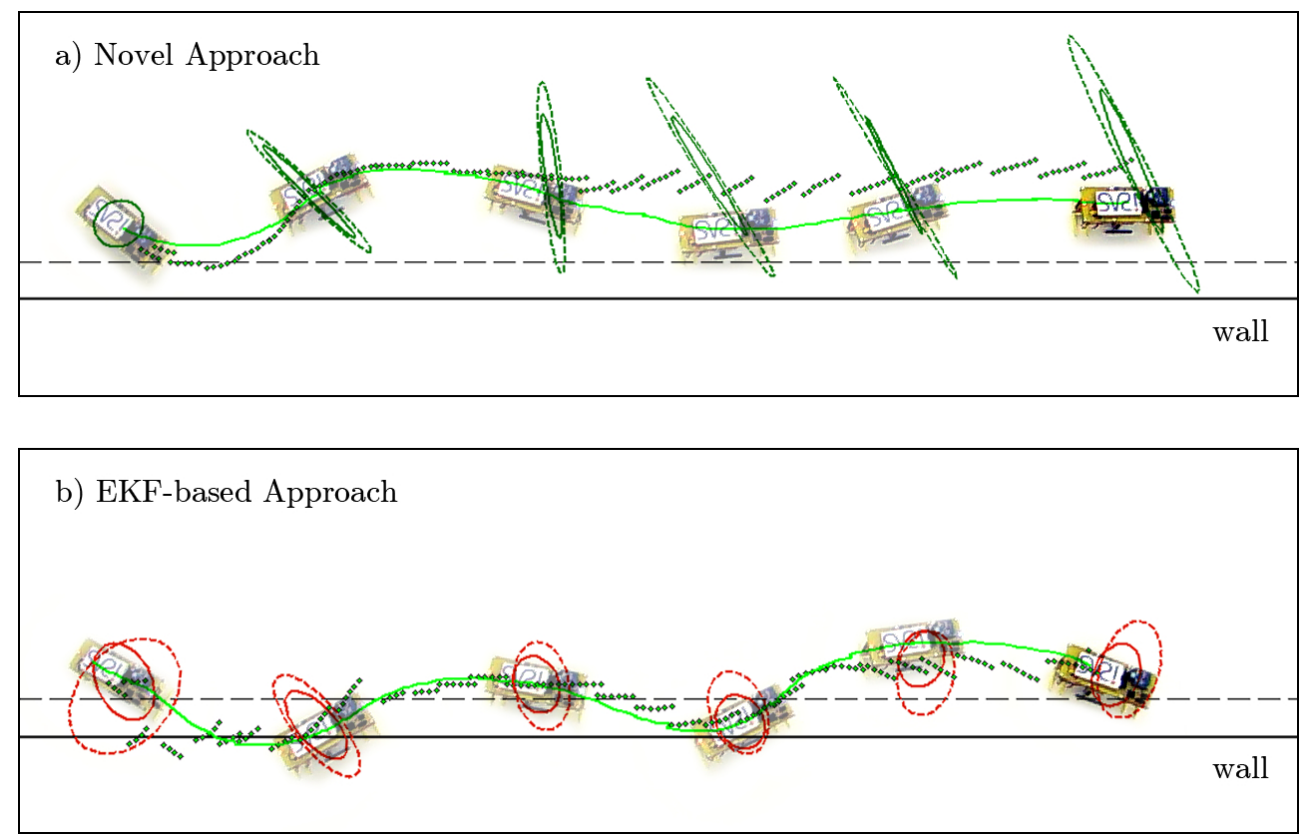

Fig. 3. Experimental runs with a) the proposed approach and b) the EKF-based NMPC method. The green solid line represents the true trajectory of the robot as determined with a high-resolution overhead camera system. The dots in subfigure a) represent the centers of the ellipsoids at each time step and the dots in subfigure b) the means. The ellipsoids in a) depict the set-valued uncertainties and the ones in b) the covariances of the estimation. In both subfigures, the dashed ellipsoids are the predicted uncertainties and the solid ellipsoids represent the uncertainties after distance measurements. For clarity, only every seventh filtering step is depicted. The figures clearly demonstrate that control employing our novel approach manages to keep the robot in a safety distance at all times, while the EKF-based control leads to close distances to the wall or even crashes.

to search for closed-form solutions of the novel combined reward function or simple parametrizations of the set of reward functions for specific scenarios.

\section{ACKNOWLEDGMENT}

This work was partially supported by the German Research Foundation (DFG) within the Research Training Group GRK 1194 "Self-organizing Sensor-ActuatorNetworks". The authors would like to thank Christof Chlebek for implementing and evaluating the presented concepts.

\section{REFERENCES}

[1] E. F. Camacho and C. Bordons, Model Predictive Control, 2nd ed. Springer-Verlag London Ltd., June 2004.

[2] D. P. Bertsekas, Dynamic Programming and Optimal Control, 2nd ed. Athena Scientific, Belmont, Massachusetts, U.S.A., 2000, vol. 1.

[3] D. Nikovski and M. Brand, "Non-Linear Stochastic Control in Continuous State Spaces by Exact Integration in Bellman's Equations," in Proceedings of the 2003 International Conference on Automated Planning and Scheduling, 2003, pp. 91-95.

[4] L. Blackmore and B. C. Williams, "Optimal, Robust Predictive Control of Nonlinear Systems under Probabilistic Uncertainty Using Particles," in Proceedings of the American Control Conference (ACC 2007), vol. 17, no. 2, New York, USA, Jul. 2007.

[5] A. Bemporad and A. Garulli, "Output-Feedback Predictive Control of Constrained Linear Systems via Set-membership State Estimation," International Journal of Control, vol. 73, no. 8, pp. 655-665, May 2000.

[6] P. O. M. Scokaert and D. Q. Mayne, "Min-Max Feedback Model Predictive Control for Constrained Linear Systems," in IEEE Transactions on Automatic Control, vol. 43, no. 8, New York, 1998.

[7] R. Huang, S. C. Patwardhan, and L. T. Biegler, "Robust Extended Kalman Filter Based Nonlinear Model Predictive Control Formulation," in Proceedings of the 2009 IEEE Conference on Decision and Control (CDC 2009), Shanghai, 2009.

[8] F. C. Schweppe, Uncertain Dynamic Systems. Prentice-Hall, 1973.
[9] A. Kurzhanski and I. Vályi, Ellipsoidal Calculus for Estimation and Control. Birkhäuser, 1997.

[10] M. Cannon, B. Kouvaritakis, and X. Wu, "Model Predictive Control for Systems with Stochastic multiplicative Uncertainty and Probabilistic Constraints," Automatica, vol. 45, no. 1, pp. 167 - 172, 2009.

[11] M. Cannon, B. Kouvaritakis, and D. Ng, "Probabilistic Tubes in Linear Stochastic Model Predictive Control," Systems \& Control Letters, vol. 58, no. 10-11, pp. $747-753,2009$.

[12] F. Weissel, M. F. Huber, and U. D. Hanebeck, "Test-Environment based on a Team of Miniature Walking Robots for Evaluation of Collaborative Control Methods," in Proceedings of the 2007 IEEE/RSJ International Conference on Intelligent Robots and Systems (IROS 2007), San Diego, California, Nov. 2007, pp. 2474-2479.

[13] I. Levi, The Enterprise of Knowledge. MIT Press, 1983.

[14] D. R. Morrell and W. C. Stirling, "Set-Valued Filtering and Smoothing," IEEE Transactions on Systems, Man and Cybernetics, vol. 21, no. 1, pp. 184-193, Jan. 1991.

[15] B. Noack, V. Klumpp, D. Brunn, and U. D. Hanebeck, "Nonlinear Bayesian Estimation with Convex Sets of Probability Densities," in Proceedings of the 11th International Conference on Information Fusion (Fusion 2008), Cologne, Germany, Jul. 2008, pp. 1-8.

[16] B. Noack, V. Klumpp, and U. D. Hanebeck, "State Estimation with Sets of Densities considering Stochastic and Systematic Errors," in Proceedings of the 12th International Conference on Information Fusion (Fusion 2009), Seattle, Washington, July 2009.

[17] D. R. Morrell and W. C. Stirling, "An Extended Set-Valued Kalman Filter," in 3rd International Symposium on Imprecise Probabilities and Their Applications (ISIPTA), 2003, pp. 396-407.

[18] F. Weissel, M. F. Huber, and U. D. Hanebeck, "A Nonlinear Model Predictive Control Framework Approximating Noise Corrupted Systems with Hybrid Transition Densities," in Proceedings of the 2007 IEEE Conference on Decision and Control (CDC 2007), New Orleans, Louisiana, Dec. 2007, pp. 3661-3666.

[19] F. Weissel, T. Schreiter, M. F. Huber, and U. D. Hanebeck, "Stochastic Model Predictive Control of Time-Variant Nonlinear Systems with Imperfect State Information," in Proceedings of the 2008 IEEE International Conference on Multisensor Fusion and Integration for Intelligent Systems (MFI 2008), Seoul, Republic of Korea, Aug. 2008, pp. 40-46. 